\title{
Far-Field Pattern Reconstruction from Near-Field Data Collected via a Nonconventional Plane-Rectangular Scanning: Experimental Testing
}

\author{
Francesco D'Agostino, ${ }^{1}$ Ilaria De Colibus, ${ }^{2}$ Flaminio Ferrara, ${ }^{1}$ Claudio Gennarelli, ${ }^{1}$ \\ Rocco Guerriero, ${ }^{1}$ and Massimo Migliozzi ${ }^{1}$ \\ ${ }^{1}$ Dipartimento di Ingegneria Industriale, Università di Salerno, Via Giovanni Paolo II, 132-84084 Fisciano (Salerno), Italy \\ ${ }^{2}$ Selex ES, Via Giulio Cesare, 105-80070 Bacoli (Naples), Italy \\ Correspondence should be addressed to Claudio Gennarelli; gennar@diiie.unisa.it
}

Received 20 January 2014; Revised 27 March 2014; Accepted 28 March 2014; Published 15 April 2014

Academic Editor: Lorenzo Crocco

Copyright (c) 2014 Francesco D’Agostino et al. This is an open access article distributed under the Creative Commons Attribution License, which permits unrestricted use, distribution, and reproduction in any medium, provided the original work is properly cited.

\begin{abstract}
This paper deals with the experimental validation of an efficient near-field-far-field (NF-FF) transformation using the planar widemesh scanning (PWMS). Such a nonconventional plane-rectangular scanning technique is so named, since the sample grid is characterized by meshes wider and wider when going away from the center, and makes it possible to lower the number of needed measurements, as well as the time required for the data acquisition when dealing with quasi-planar antennas. It relies on the use of the nonredundant sampling representations of electromagnetic fields which employ an oblate ellipsoid or a surface formed by two circular "bowls" with the same aperture diameter but eventually different bending radii to shape a quasi-planar antenna. A twodimensional optimal sampling interpolation formula allows the reconstruction of the NF data at any point on the measurement plane and, in particular, at those required by the classical NF-FF transformation with the conventional plane-rectangular scanning. The measurements, performed at the planar NF facility of the antenna characterization laboratories of Selex ES, have confirmed the effectiveness of this innovative scanning also from the experimental viewpoint.
\end{abstract}

\section{Introduction}

As well known, the evaluation of the radiation characteristics represents a crucial step in the design of an antenna to verify whether the initial specifications are met and the antenna may be effectively employed for the desired application. The simplest measurement method consists in the direct far-field (FF) measurement. In order to perform accurate antenna measurements, the influence of uncontrollable environment conditions (rain, snow, electromagnetic interferences, etc.) can be reduced as much as possible by carrying out the measurements in anechoic chambers, which, making negligible the reflections from the walls, ensure free-space propagation conditions. Unfortunately, the FF distance requirements cannot be practically satisfied when dealing with antennas having large or even medium electrical sizes, so that only near-field (NF) measurements can be performed. Accordingly, the FF pattern can be determined by postprocessing the acquired NF data via NF-FF transformations [1-6]. The NF measurements have also the advantage to allow the determination of complete pattern and polarization characteristics and provide the necessary information for obtaining the radiating field on the antenna surface needed for holographic diagnostic purposes [7].

Two main kinds of NF-FF transformation techniques can be distinguished to characterize the FF pattern of an antenna under test (AUT) from NF measurements: the ones based on the equivalent electromagnetic (EM) sources reconstruction methods and those using a modal expansion approach.

In the former, the equivalent currents on a selected surface enclosing the antenna are evaluated by solving a set of integral equations relating these (unknown) currents to the NF data acquired on the scanning surface [8-13]. Once these equivalent currents have been determined, according to 
Love's equivalence principle, it is possible to obtain the field at any point outside the equivalent source domain and, then, to evaluate the FF pattern.

In the latter, the measured NF data are transformed into FF patterns by using an expansion of the field of the AUT in terms of modes, namely, a complete set of solutions of the vector wave equation in the region outside the antenna. Plane, cylindrical, or spherical waves are generally used. The type of employed modal expansion determines the kind of the NF scanning surface, which accordingly will be a plane, a cylinder, or a sphere. The orthogonality properties of the modes on such surfaces are then exploited to obtain the modal expansion coefficients, which allow the reconstruction of the AUT far field. The development and spreading of NFFF transformation techniques using planar [14, 15], cylindrical [16], or spherical [17] scanning is justified from the fact that each has its own particular advantages, depending on the AUT and measurement requirements.

Among them, that employing the standard planerectangular scanning is the most simple and efficient one from the NF data acquisition, analytical, and computational viewpoints. Its development stems from the plane wave spectrum representation of EM fields [18]. As a matter of fact, the AUT far field is related to the two-dimensional Fourier transforms of the tangential components of the AUT field. In any practical instance, the measurements are carried out by a real (not ideal) probe in two orientations and, accordingly, its directional effects must be properly taken into account in evaluating the AUT far field via the NF-FF transformation. The related probe corrected formulas can be derived by applying the Lorentz reciprocity theorem as in $[14,15]$ or by employing the scattering matrix formulation [19]. The main disadvantage of the NF-FF transformation technique with plane-rectangular scan is that the pattern can be reconstructed only in a cone with an apex angle less than $180^{\circ}$ without repeating the measurements. Thus, it is particularly suitable for highly directive antennas which radiate pencil beam patterns well within the solid angle specified by the edges of the AUT and those of the scanning area. Even though the alignment procedures are easy to implement and the AUT remains stationary during testing, it requires the sampling rate be constant along the scanner axes and the distance between two consecutive samples be bounded by $\lambda / 2, \lambda$ being the wavelength corresponding to the working frequency. Accordingly, the a priori information about the AUT size has no influence upon the sampling rate.

On the contrary, such information has been properly taken into account in the NF-FF transformation techniques using the innovative planar wide-mesh scanning (PWMS) $[20,21]$, which rely on the nonredundant sampling representations of EM fields $[22,23]$. Such a nonconventional planerectangular scanning is characterized (see Figure 1) by a sample grid having linear spacing between two consecutive lines, along $x$ and $y$, which increases and exceeds $\lambda / 2$ as their distance from the center of the scanning plane increases. These NF-FF transformation techniques employ a two-dimensional optimal sampling interpolation (OSI) formula which allows the effective reconstruction of the NF data required by the NF-FF transformation with the classical plane-rectangular

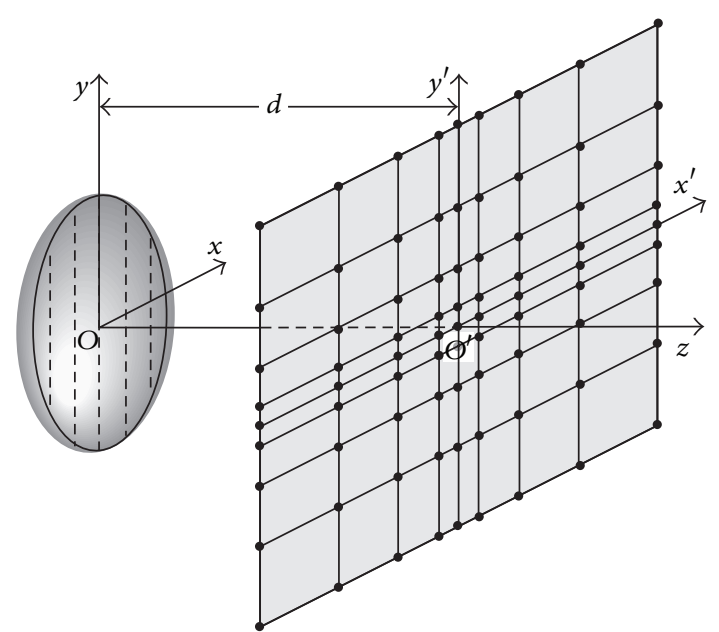

FIGURE 1: Planar wide-mesh scanning for a quasi-planar antenna.

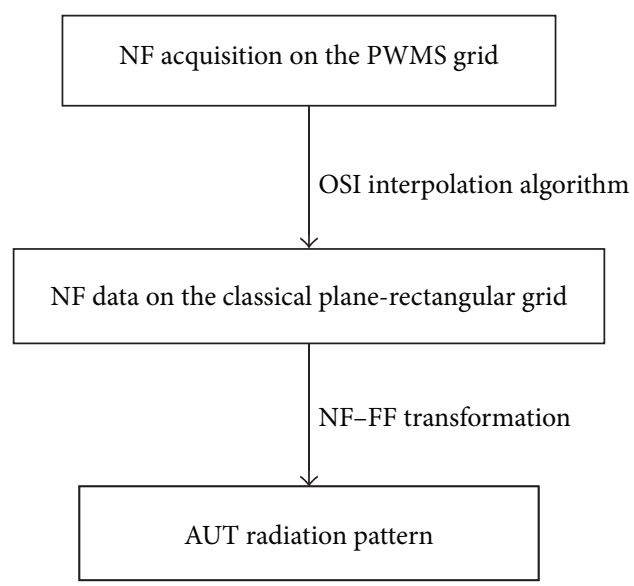

Figure 2: Flowchart of the algorithm.

scanning $[14,15]$ from those collected through PWMS. In particular, a spherical source modelling has been adopted in [20], whereas an oblate ellipsoid or a surface formed by two circular "bowls" with the same aperture diameter but eventually different lateral bends (two-bowl modelling) has been employed in [21] to shape the AUT. These last modellings significantly lower the number of required NF data when dealing with quasi-planar antennas and allow to consider a measurement plane at a distance smaller than one half the antenna maximum size, thus lowering the error related to the truncation of the scanning region. Note that the practical implementation of the PWMS requires no drastic changes in an existing plane-rectangular NF facility, being only necessary to properly modify the software controlling the linear movements of the positioning systems and to introduce a further data processing procedure between the data acquisition and the standard NF-FF transformation (see flowchart in Figure 2).

It is worthy to note that an adaptive sampling technique for reducing the planar NF data needed for reconstructing the AUT radiation pattern has been developed in [24]. According 
to this approach, the NF data are adaptively acquired on rectangular rings and, based on a given decision function, the measurement system concentrates the acquisition on the strongly changing NF regions while skipping the measurement points from the smoothly varying ones. The FF pattern is then obtained from the so-collected NF data via the multilevel plane wave based NF-FF transformation algorithm [25].

The aim of this paper is to provide the experimental assessment of the NF-FF transformations with PWMS using both the two-bowl modelling and the oblate ellipsoidal one to shape a quasi-planar AUT. However, such a validation, due to the unavailability of an $x-y$ scanner, cannot be performed at the UNISA Antenna Characterization Lab wherein, on the contrary, it has been possible to carry out several measurement campaigns assessing the validity [26-34] of the nonredundant NF-FF transformation techniques using cylindrical and spherical scanning geometries. Fortunately, a recent research agreement with Selex ES, a Finmeccanica company, whose antenna characterization laboratories are provided with planar NF facilities, has offered the opportunity for this experimental validation.

\section{NF-FF Transformation with the Nonconventional Plane-Rectangular Scan}

In the plane-rectangular scanning, the probe mounted on an $x-y$ positioner acquires the NF data on a plane located at a distance $d$ from the AUT center, which is assumed as origin of the Cartesian coordinate system $x, y, z$ (see Figure 1). When considering a nondirective probe, the measured voltage has the same effective spatial bandwidth of the field radiated by the AUT and, accordingly, the application of the theoretical results on the nonredundant representations of EM fields [22] enables us to approximate the voltage measured by such a kind of probe with a proper bandlimited function. As a matter of fact, when a proper surface $\Sigma$ is adopted for modelling an electrically large AUT and a proper parameterization $\xi$ is employed to describe an observation curve on a plane external to $\Sigma$, then the "reduced voltage" $\widetilde{V}(\xi)=V(\xi) e^{j \gamma(\xi)}$, $\gamma(\xi)$ being a proper phase function, is well approximated by a spatially bandlimited function.

The resulting bandlimitation error, which exhibits a steplike behaviour [22, 23], becomes negligible as the bandwidth exceeds a critical value $W_{\xi}$. As a consequence, it can be effectively controlled by choosing a bandwidth equal to $\chi^{\prime} W_{\xi}$, $\chi^{\prime}>1$ being an excess bandwidth factor [22].

When $C$ is a radial line as the $x^{\prime}$ (or $y^{\prime}$ ) axis, by denoting with $s_{1,2}^{\prime}$ the arclength coordinates of the two tangency points $P_{1,2}$ on $C^{\prime}$ (intersection curve between the meridian plane passing through the observation point $P$ and $\Sigma$ ), with $R_{1,2}$ the distances from $P$ to $P_{1,2}$ and choosing

$$
W_{\xi}=\frac{\beta \ell^{\prime}}{2 \pi}
$$

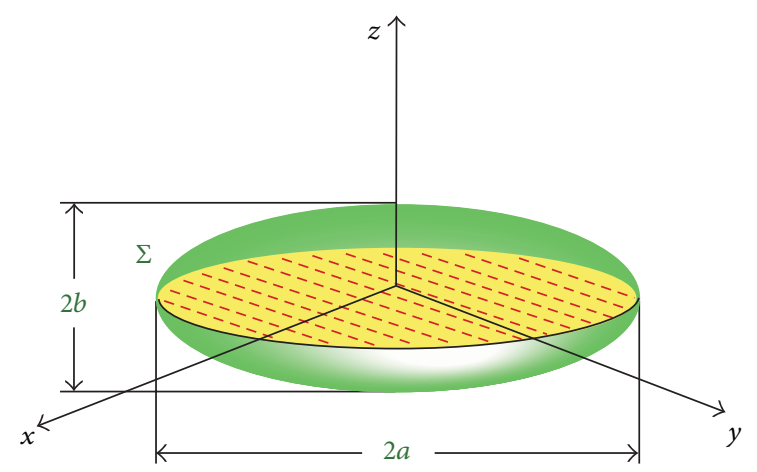

FIGURE 3: Oblate ellipsoidal modelling of the AUT.

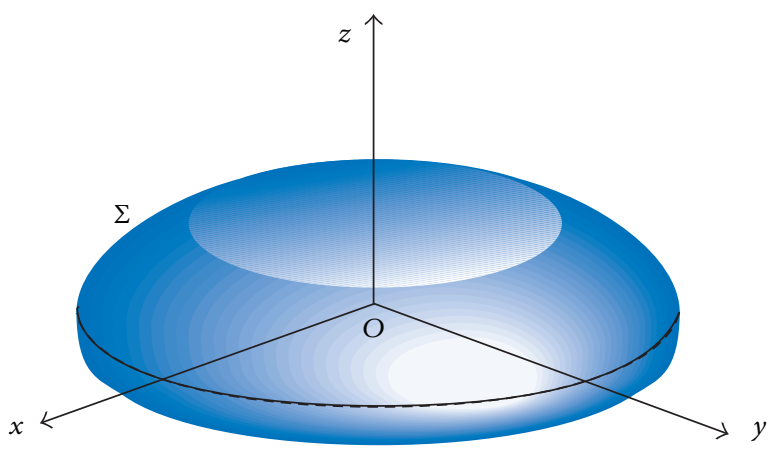

FIgURE 4: Two-bowl modelling of the AUT.

$\ell^{\prime}$ being the length of $C^{\prime}$ and $\beta$ being the wavenumber, we obtain [22]

$$
\begin{aligned}
& \xi=\left(\frac{\pi}{\ell^{\prime}}\right)\left[R_{1}-R_{2}+s_{1}^{\prime}+s_{2}^{\prime}\right], \\
& \gamma=\left(\frac{\beta}{2}\right)\left[R_{1}+R_{2}+s_{1}^{\prime}-s_{2}^{\prime}\right] .
\end{aligned}
$$

According to the strategy defined by the nonredundant representations, the number of the needed NF data can be reduced more and more by employing effective antenna modellings able to fit better and better an AUT characterized by a quasi-planar geometry. Therefore, it is convenient to choose the surface $\Sigma$, enclosing it, coincident [21] with an oblate ellipsoid (see Figure 3) or a surface formed by two circular "bowls" with the same aperture diameter but eventually different bending radii (see Figure 4), named two-bowl modelling. Since relations (1)-(3) depend on the particular choice of $\Sigma$ enclosing the AUT and the observation point, their expressions change accordingly (see Appendices A and B).

It is worthy to note that, besides the remarkable reduction of the NF data, these AUT modellings, in spite of the spherical one, allow to consider a measurement plane located at a distance less than one half the antenna maximum size, thus lowering the error related to the truncation of the scanning region.

In order to factorize the two-dimensional interpolation scheme into one-dimensional OSI expansions along lines 
$[20,21]$, it is mandatory to adopt the same parameter $\xi$ (or $\eta$ ) given by (2) for describing all lines parallel to the $x^{\prime}\left(y^{\prime}\right)$ axis. Therefore, the samples spacing on them is the same of the $x^{\prime}$ $\left(y^{\prime}\right)$ axis (Figure 1). This corresponds to the use of a parameter that does not make the local bandwidth constant, but, since such a parameter is always less than or equal to $W_{\xi}$, the number of samples is greater than that required by a rigorous application of a nonredundant sampling representation and no further representation error is introduced [21]. The soobtained grid has meshes wider and wider when going away from the center. For what concerns the phase function $\gamma$, expression (3) can be still adopted.

It must be stressed that, since all scanning lines have the same linear sampling rate, the acquisition points are aligned also along lines perpendicular to the scanning ones (see Figure 1). Accordingly, the acquisition process is just analogous to the classical plane-rectangular one and can be performed by using the same devices and positioning systems. Only the software code devoted to controlling the NF data acquisition must be changed.

According to the above described nonredundant sampling representation, the reduced voltage can be evaluated at any observation point $P$ on the plane, via the following twodimensional OSI expansion [21]:

$$
\begin{aligned}
& V(\xi, \eta) \\
& =\widetilde{V}(\xi, \eta) e^{-j \gamma\left(x^{\prime}, y^{\prime}\right)} \\
& =e^{-j \gamma\left(x^{\prime}, y^{\prime}\right)} \\
& \quad \times \sum_{n=n_{0}-q+1}^{n_{0}+q}\left\{\Omega_{N}\left(\xi-\xi_{n}, \bar{\xi}\right) D_{N^{\prime \prime}}\left(\xi-\xi_{n}\right)\right. \\
& \quad \times \sum_{m=m_{0}-p+1}^{m_{0}+p} \widetilde{V}\left(\xi_{n}, \eta_{m}\right) \Omega_{N}\left(\eta-\eta_{m}, \bar{\eta}\right) \\
& \left.\times D_{N^{\prime \prime}}\left(\eta-\eta_{m}\right)\right\},
\end{aligned}
$$

where $2 q$ and $2 p$ are the numbers of samples $\widetilde{V}\left(\xi_{n}, \eta_{m}\right)$ considered in the interpolations along $\xi$ and $\eta$, respectively,

$$
\begin{gathered}
\xi_{n}=n \Delta \xi=\frac{2 n \pi}{\left(2 N^{\prime \prime}+1\right)} ; \\
\eta_{m}=m \Delta \eta=\frac{2 m \pi}{\left(2 N^{\prime \prime}+1\right)} ; \\
n_{0}=\operatorname{Int}\left(\frac{\xi}{\Delta \xi}\right) ; \quad m_{0}=\operatorname{Int}\left(\frac{\eta}{\Delta \eta}\right) ; \\
\bar{\xi}=q \Delta \xi ; \quad \bar{\eta}=p \Delta \eta ; \\
N^{\prime \prime}=\operatorname{Int}\left(\chi N^{\prime}\right)+1 ; \quad N^{\prime}=\operatorname{Int}\left(\chi^{\prime} W_{\xi}\right)+1 ;
\end{gathered}
$$

$\operatorname{Int}(x)$ denotes the integer part of $x$ and $\chi>1$ is the oversampling enlargement factor [21]. Moreover,

$$
\begin{aligned}
D_{N^{\prime \prime}}(x) & =\frac{\sin \left[\left(2 N^{\prime \prime}+1\right) x / 2\right]}{\left(2 N^{\prime \prime}+1\right) \sin (x / 2)}, \\
\Omega_{N}(x, \bar{x}) & =\frac{T_{N}\left[2 \cos ^{2}(x / 2) / \cos ^{2}(\bar{x} / 2)-1\right]}{T_{N}\left[2 / \cos ^{2}(\bar{x} / 2)-1\right]}
\end{aligned}
$$

are the Dirichlet and Tschebyscheff sampling functions, respectively $[21,22]$, and $T_{N}(\cdot)$ is the Tschebyscheff polynomial of degree $N=N^{\prime \prime}-N^{\prime}$.

The OSI formula (4) can be employed to recover the NF data at the points required by the classical probecompensated NF-FF transformation with plane-rectangular scanning [14].

\section{Experimental Testing}

This section is devoted to show some experimental results assessing the effectiveness of the described NF-FF transformation technique with PWMS suitable for quasi-planar antennas.

The measurement campaign has been performed in the anechoic chamber $(13 \mathrm{~m} \times 10 \mathrm{~m} \times 7 \mathrm{~m}$ sized $)$ of the antenna characterization laboratories of Selex ES, equipped with the planar NF facility. Such a facility is realized with an advanced three-dimensional scanner, formed by a mechanical arm able to move along the $z$-axis, which is mounted on a vertical slide ( $y^{\prime}$-axis of the reference system) moving along the $x^{\prime}$-axis on a granite plinth. It allows the scanning on a plane having maximum dimensions of $6 \mathrm{~m} \times 5 \mathrm{~m}$. The AUT is located in front of the scanner using a proper support, while the probe, mounted at the end of the arm, acquires amplitude and phase of the NF data when the AUT works in the transmitting (TX) mode. The facility allows the characterization of antennas working also in the receiving ( $\mathrm{RX})$ mode, such as the active antennas of the latest generation, equipped with integrated receivers. Moreover, the test equipment is able to perform measurements both in the RF band, from $1 \mathrm{GHz}$ to $12 \mathrm{GHz}$, and in the IF one, up to $60 \mathrm{MHz}$. It allows both to supply or receive the RF/IF signals of the RX/TX measurements and also to manage the commands for driving the antenna. The hardware implementing the test equipment is realized by standard PXI cards inserted in chassis, thus ensuring the modularity of the measurement system. Proper software interfaces are devoted to control the movements of the scanner, to collect the data on the wanted grid, and to perform the needed postprocessing of the acquired NF data. This last module has been recently upgraded, in collaboration with the University of Salerno, with an enhanced version of the NF-FF transformations with the classical plane-rectangular scanning and the nonconventional PWMS one.

In order to reduce the overall acquisition time, both the classical and nonconventional scanning modules controlling the linear movements of the positioning systems have implemented in such a way that the motions in the vertical direction are alternated. 


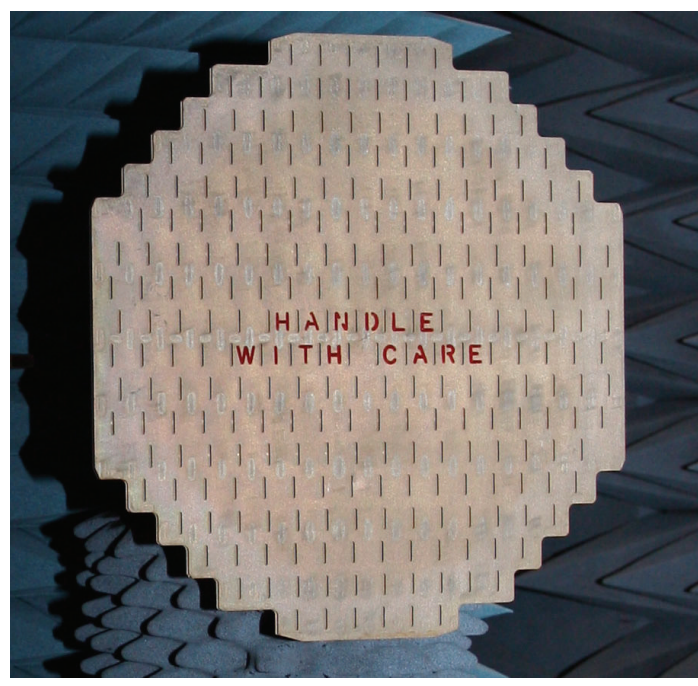

FIGURE 5: Photo of the X-band flat plate slot array.

The AUT employed in the experimental testing is shown in Figure 5. It is an X-band flat plate slot array of Rantec Microwave Systems Inc., having a diameter of about $46 \mathrm{~cm}$, located at $z=0$, and the measurement plane is at $z=$ $d=22.4 \mathrm{~cm}$ (see Figure 1). The optimal working frequencies of the considered AUT are 9.2, 9.3, 9.4, and 9.5 GHz. The reported results are relevant to 9.3 and $9.4 \mathrm{GHz}$.

An accurate alignment procedure, performed with a quadrant detector and a laser, has been made in order to ensure that the scanning plane is nominally parallel to the slot plane of the AUT. Moreover, the mounting structure supporting the probe, an open-ended WR-90 rectangular waveguide, has been well covered with absorbers to minimize the scattering toward the AUT.

Since a multifrequency acquisition is planned, the parameters relevant to the considered AUT modellings are defined by referring to the highest working frequency. The AUT has been fitted by a two-bowl modelling having parameters $a=$ $24 \mathrm{~cm}, c=8 \mathrm{~cm}$, and $c^{\prime}=7.5 \mathrm{~cm}$ and by an oblate ellipsoid with major and minor semiaxes equal to $26.5 \mathrm{~cm}$ and $8.2 \mathrm{~cm}$, respectively. It is worthy to note that these choices have been made in order to consider the same number of PWMS lines $(51 \times 51)$ for covering a measurement plane $3 \mathrm{~m} \times 3 \mathrm{~m}$ sized, the same extension considered in the classical plane-rectangular scanning case.

In order to assess the effectiveness of the two-dimensional OSI expansion (4), the reconstructed probe voltage on the horizontal line of the measurement plane at $y^{\prime}=10 \mathrm{~cm}$ has been compared with that directly measured on the same line. Figure 6 shows this comparison when the AUT works at 9.3 GHz and the two-bowl modelling is adopted, whereas the analogous result, when considering the ellipsoidal modelling and the AUT working at $9.4 \mathrm{GHz}$, is reported in Figure 7. Obviously, analogous results have been obtained for the other two considered working frequencies.

As can be seen, the reconstructions are everywhere accurate save for the peripheral zone where the directly measured voltages result to be more oscillating as compared to the

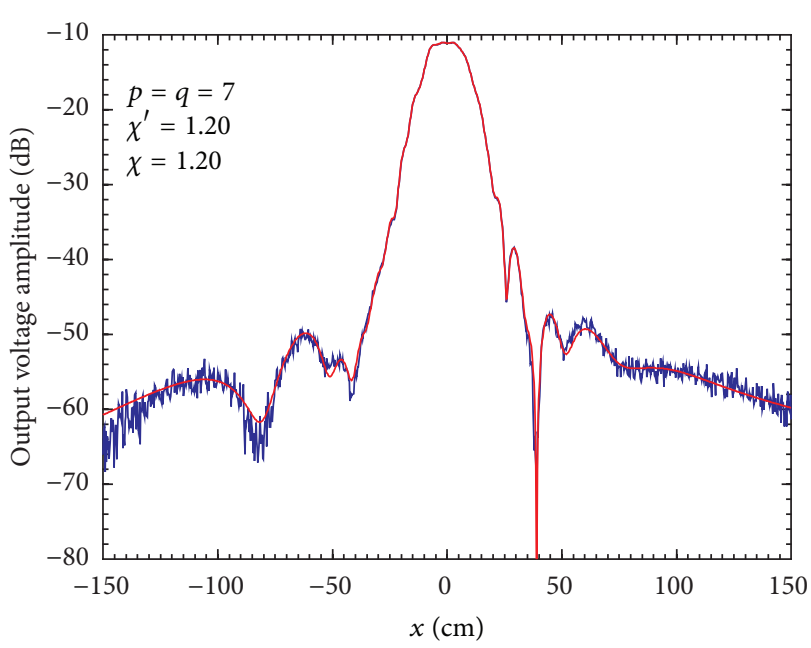

Figure 6: AUT @ 9.3 GHz. Amplitude of the probe voltage on the line at $y^{\prime}=10 \mathrm{~cm}$. Blue line: measured. Red line: recovered from NF data acquired via PWMS when using the two-bowl modelling.

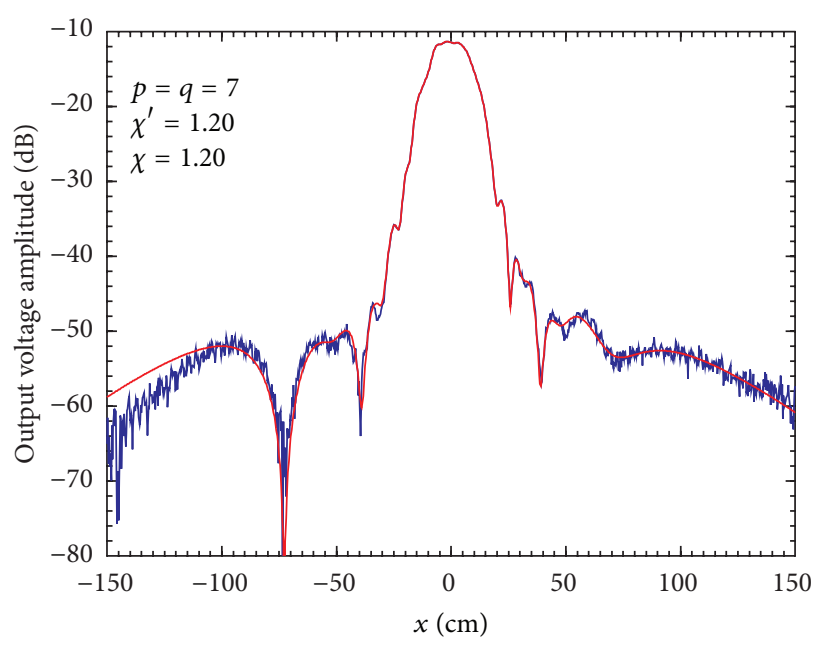

Figure 7: AUT @ 9.4 GHz. Amplitude of the probe voltage on the line at $y^{\prime}=10 \mathrm{~cm}$. Blue line: measured. Red line: recovered from NF data acquired via PWMS when using the oblate ellipsoidal modelling.

reconstructed ones, which exhibit a smoother behaviour. This is due to the low pass filtering properties of the interpolation functions, which cut away the spatial harmonics related to the noise sources outside the AUT spatial bandwidth. Moreover, the voltage reconstruction obtained when using the oblate ellipsoidal modelling seems to degrade in the peripheral zone with respect to that achieved when employing the two-bowl modelling. This occurs since, having used the same number of PWMS lines for both of the examples, the scanning area coverage attainable by employing the two-bowl modelling is greater than that achievable by the oblate ellipsoidal one. In such a case, the reconstruction can be improved if the AUT modelling parameters are set in such a way to take into account an increased number of PWMS lines to cover the same measurement plane. It must be stressed that the use 


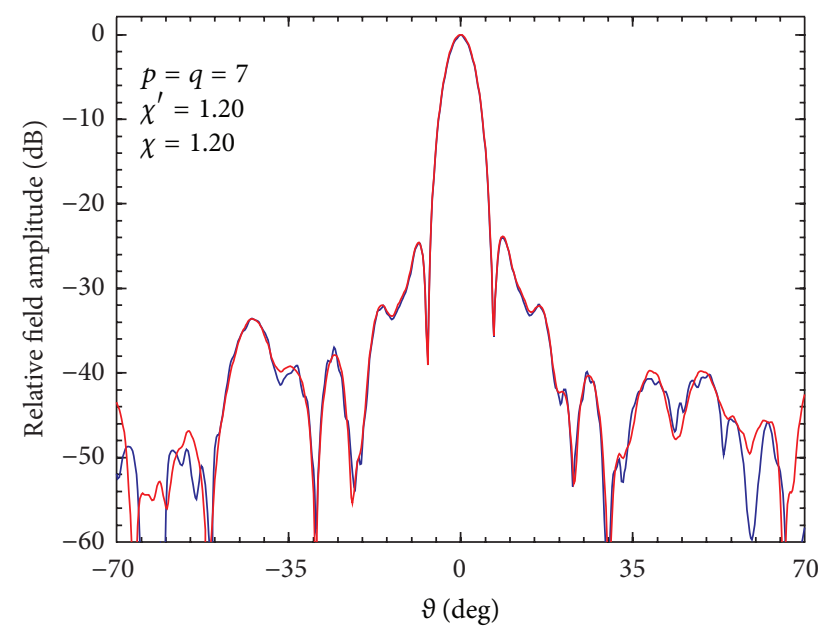

FIGURE 8: AUT @ 9.3 GHz. E-plane pattern. Blue line: reference. Red line: recovered from NF data acquired via the PWMS when using the two-bowl modelling.

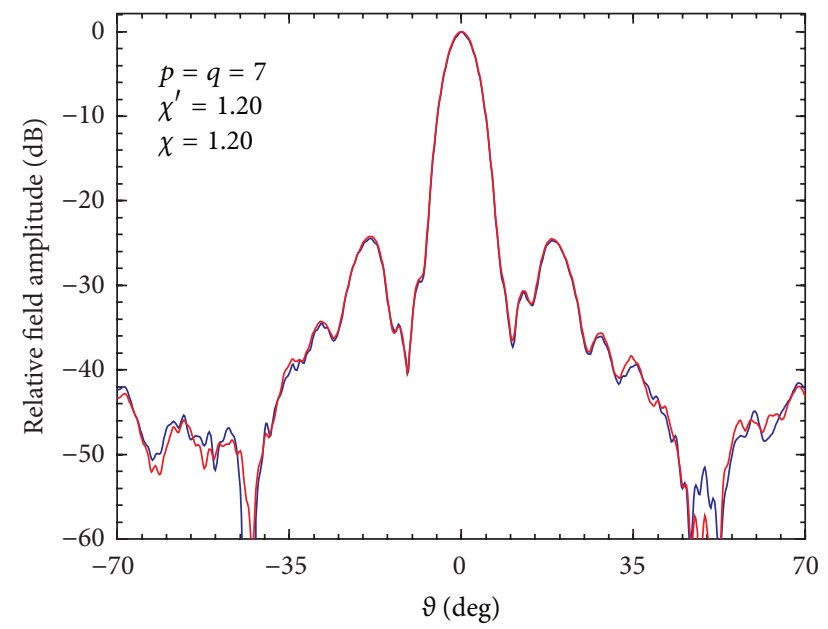

Figure 9: AUT @ 9.3 GHz. $H$-plane pattern. Blue line: reference. Red line: recovered from NF data acquired via the PWMS when using the two-bowl modelling.

of the OSI expansions, instead of the cardinal series ones, improves the robustness of the technique when dealing with noisy data, since it prevents the propagation of the errors affecting the data from higher to lower voltage regions.

At last, the FF patterns in the principal planes $E$ and $H$ reconstructed from the NF set of measurements acquired through the PWMS scan are compared in Figures 8-11 with those (references) obtained from the NF data directly measured on the classical plane-rectangular grid. In particular, Figures 8 and 9 show those relevant to the AUT at $9.3 \mathrm{GHz}$ when using the two-bowl modelling, whereas Figures 10 and 11 refer to the AUT at $9.4 \mathrm{GHz}$ when considering the ellipsoidal modelling. In all the cases, the software package performing the classical NF-FF transformation has been employed to get the FF reconstructions. Obviously, the PWMS data have been interpolated via the two-dimensional OSI expansion (4) to recover the needed classical redundant

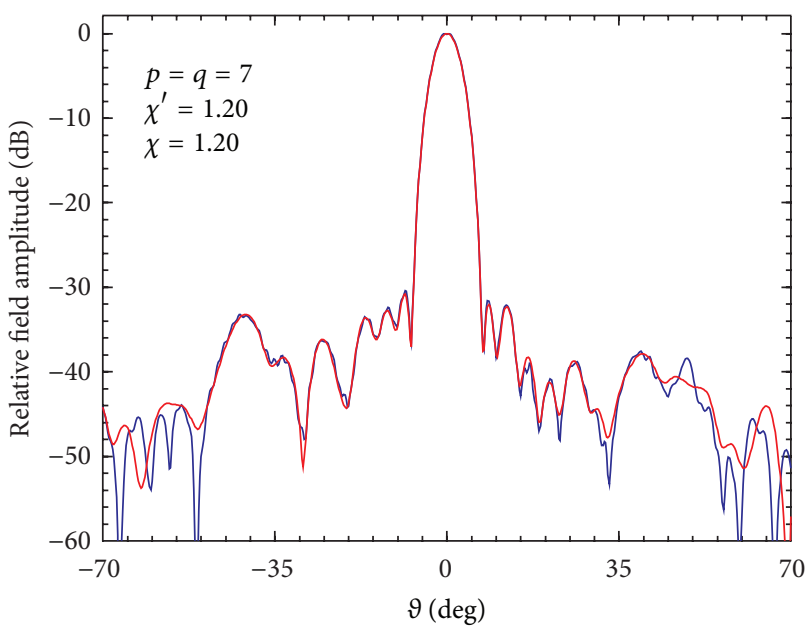

Figure 10: AUT @ $9.4 \mathrm{GHz}$. E-plane pattern. Blue line: reference. Red line: recovered from NF data acquired via the PWMS when using the ellipsoidal modelling.

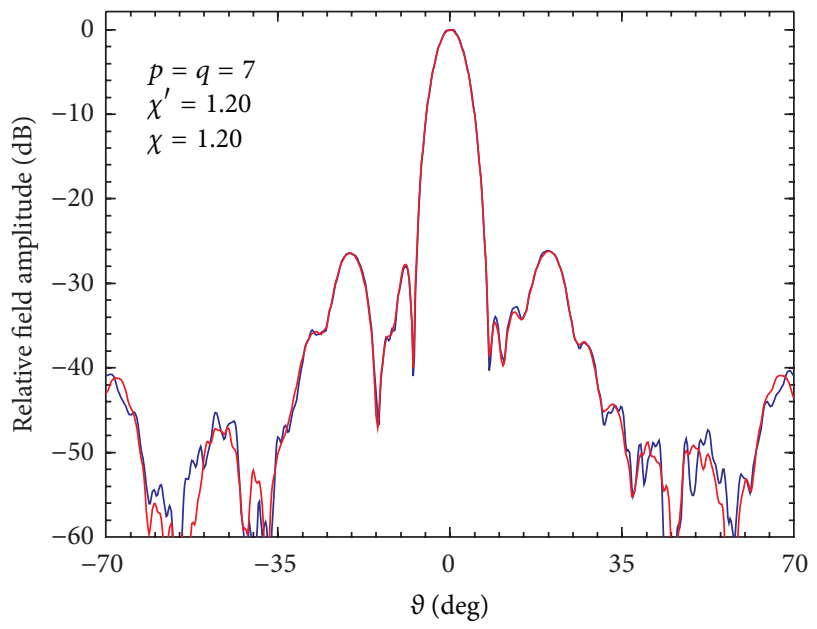

FIGURE 11: AUT @ 9.4 GHz. H-plane pattern. Blue line: reference. Red line: recovered from NF data acquired via the PWMS when using the ellipsoidal modelling.

ones. As can be seen, the reconstructions are very accurate save for the far-out sidelobes (below about $-45 \mathrm{~dB}$ ) region, wherein the error is caused by the noise and the residual reflections from the anechoic chamber walls. Quite analogous results (not reported here for brevity) have been obtained for the other two considered working frequencies. Also the FF reconstructions confirm the overall effectiveness of the approach.

It must be stressed, for sake of comparison, that the number of PWMS data for covering the considered measurement plane is 2601 against the 40401 ones needed by the classical NF-FF transformation with plane-rectangular scanning [14]. As regards the measurement time, the PWMS acquisition has taken about an hour and a half, whereas the classical planerectangular one about twenty-two hours. 


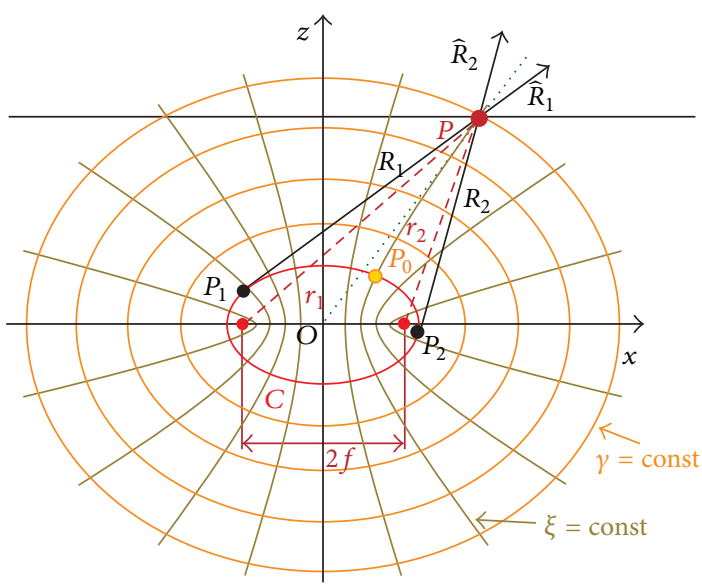

(a)

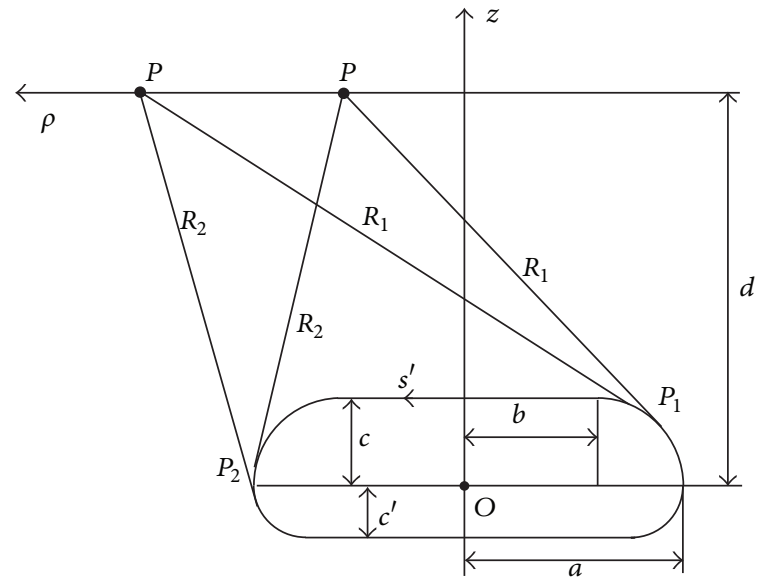

(b)

FIgURE 12: (a) Relevant to the oblate ellipsoidal modelling. (b) Relevant to the two-bowl modelling.

\section{Conclusions}

An experimental validation of the NF-FF transformations with PWMS, suitable for quasi-planar antennas, has been provided in this paper. The experimental tests have been possible thanks to the research agreement with Selex ES, whose antenna characterization laboratories are equipped with planar NF facilities. The very good results achieved both in the near-field and in the far-field reconstructions confirm also from the experimental viewpoint the validity of such a nonconventional scanning, which allows a drastic NF data reduction, as well as measurement time saving, without losing the accuracy of that using the conventional scan and without requiring drastic changes in an existing plane-rectangular NF facility.

\section{Appendices}

\section{A. Relevant to the Oblate Ellipsoidal AUT Modelling}

When considering the AUT as enclosed in the smallest oblate ellipsoid having major and minor semiaxes equal to $a$ and $b$ (see Figure 12(a)), relations (1)-(3) can be rewritten as follows [21]:

$$
\begin{aligned}
W_{\xi} & =\frac{4 a}{\lambda} E\left(\frac{\pi}{2} \mid \varepsilon^{2}\right), \\
\xi & =\frac{\pi}{2}\left[\frac{E\left(\sin ^{-1} u \mid \varepsilon^{2}\right)}{E\left((\pi / 2) \mid \varepsilon^{2}\right)}\right], \\
\gamma & =\beta a\left[v \sqrt{\frac{v^{2}-1}{v^{2}-\varepsilon^{2}}}-E\left(\cos ^{-1} \sqrt{\frac{1-\varepsilon^{2}}{v^{2}-\varepsilon^{2}}} \mid \varepsilon^{2}\right)\right],
\end{aligned}
$$

where $E(\cdot \mid \cdot)$ is the elliptic integral of second kind, $\varepsilon=$ $f / a$ is the ellipsoid eccentricity, $2 f$ is its focal distance, and $u=\left(r_{1}-r_{2}\right) / 2 f$, and $v=\left(r_{1}+r_{2}\right) / 2 a$ are the elliptic coordinates, with $r_{1,2}$ being the distances from $P$ to the foci.

\section{B. Relevant to the Two-Bowl AUT Modelling}

When adopting the two-bowl modelling (Figure 12(b)), the bandwidth $W_{\xi}$, the optimal expressions for the phase factor $\gamma$, and parameterization $\xi$ relevant to a radial line are again given by relations (1)-(3). In such a case [21],

$$
\ell^{\prime}=2\left[(a-c)+\left(a-c^{\prime}\right)+\frac{\left(c+c^{\prime}\right) \pi}{2}\right]
$$

whereas the expressions of the distances $R_{1,2}$ from the observation point $P$ to the tangency points $P_{1,2}$ on $C^{\prime}$, and of their arclength coordinates $s_{1,2}^{\prime}$ change depending on the location of $P_{1,2}$ (see Figure 12(b)). In particular, when they are both located on the upper bowl $(\rho<a)$, we obtain [21]

$$
\begin{gathered}
R_{1}=\sqrt{(\rho+b)^{2}+d^{2}-c^{2}} ; \quad b=a-c, \\
s_{1}^{\prime}=-\left(b+c \alpha_{1}\right) ; \quad \alpha_{1}=\tan ^{-1}\left(\frac{R_{1}}{c}\right)-\tan ^{-1}\left[\frac{(\rho+b)}{d}\right] \\
R_{2}=\sqrt{(\rho-b)^{2}+d^{2}-c^{2}} ; \quad s_{2}^{\prime}=b+c \alpha_{2}, \\
\alpha_{2}=\tan ^{-1}\left(\frac{R_{2}}{c^{\prime}}\right)-\tan ^{-1}\left[\frac{(b-\rho)}{d}\right]
\end{gathered}
$$


When $P_{2}$ is on the lower bowl ( $\rho \geq a$ ), the expressions of $R_{1}, s_{1}^{\prime}$, and $\alpha_{1}$ are again given by relations (B.2) and (B.3), whereas the expressions of $R_{2}, s_{2}^{\prime}$, and $\alpha_{2}$ change into

$$
\begin{aligned}
& R_{2}=\sqrt{\left(\rho-b^{\prime}\right)^{2}+d^{2}-c^{\prime 2}} ; \quad b^{\prime}=a-c^{\prime}, \\
& s_{2}^{\prime}=b+\frac{c \pi}{2}+c^{\prime} \alpha_{2}, \\
& \alpha_{2}=\tan ^{-1}\left(\frac{R_{2}}{c^{\prime}}\right)-\frac{\pi}{2}+\tan ^{-1}\left[\frac{\left(\rho-b^{\prime}\right)}{d}\right] .
\end{aligned}
$$

\section{Conflict of Interests}

The authors declare that there is no conflict of interests regarding the publication of this paper.

\section{Acknowledgment}

The authors would like to express their thanks to Dr. Stefano Pitta for his priceless support in performing the testing.

\section{References}

[1] A. D. Yaghjian, "An overview of near-field antenna measurements," IEEE Transactions on Antennas and Propagation, vol. 34, no. 1 , pp. 30-45, 1986.

[2] J. Appel-Hansen, J. D. Dyson, E. S. Gillespie, and T. G. Hickman, "Antenna measurements," in The Handbook of Antenna Design, A. W. Rudge, K. Milne, A. D. Olver, and P. Knight, Eds., chapter 8, Peter Peregrinus, London, UK, 1986.

[3] E. S. Gillespie, Ed., "Special issue on near-field scanning techniques," IEEE Transactions on Antennas and Propagation, vol. 36, no. 6, pp. 727-901, 1988.

[4] M. H. Francis and R. W. Wittmann, "Near-field scanning measurements: theory and practice," in Modern Antenna Handbook, C. A. Balanis, Ed., chapter 19, John Wiley \& Sons, Hoboken, NJ, USA, 2008.

[5] C. Gennarelli, A. Capozzoli, L. Foged, J. Fordham, and D. J. van Rensburg, "Recent advances in near-field to far-field transformation techniques," International Journal of Antennas and Propagation, vol. 2012, Article ID 243203, 3 pages, 2012.

[6] M. H. Francis, Ed., "IEEE Recommended Practice for NearField Antenna Measurements," IEEE Standard 1720-2012.

[7] R. G. Yaccarino, Y. Rahmat-Samii, and L. I. Williams, "Bi-polar planar near-field measurement technique-part II: near-field to far-field transformation and holographic imaging methods," IEEE Transactions on Antennas and Propagation, vol. 42, no. 2, pp. 196-204, 1994.

[8] P. Petre and T. K. Sarkar, "Planar near-field to far-field transformation using an equivalent magnetic current approach," IEEE Transactions on Antennas and Propagation, vol. 40, no. 11, pp. 1348-1356, 1992.

[9] P. Petre and T. K. Sarkar, "Differences between modal expansion and integral equation methods for planar near-field to far-field transformation," Progress in Electromagnetics Research, vol. 12, pp. 37-56, 1996.

[10] A. Taaghol and T. K. Sarkar, "Near-field to near/far-field transformation for arbitrary near-field geometry, utilizing an equivalent magnetic current," IEEE Transactions on Electromagnetic Compatibility, vol. 38, no. 3, pp. 536-542, 1996.

[11] T. K. Sarkar and A. Taaghol, "Near-field to near/far-field transformation for arbitrary near-field geometry utilizing an equivalent electric current and MoM," IEEE Transactions on Antennas and Propagation, vol. 47, no. 3, pp. 566-573, 1999.

[12] F. Las-Heras and T. K. Sarkar, "Radial field retrieval in spherical scanning for current reconstruction and NF-FF transformation," IEEE Transactions on Antennas and Propagation, vol. 50, no. 6, pp. 866-874, 2002.

[13] F. Las-Heras, M. R. Pino, S. Loredo, Y. Alvarez, and T. K. Sarkar, "Evaluating near-field radiation patterns of commercial antennas," IEEE Transactions on Antennas and Propagation, vol. 54, no. 8, pp. 2198-2207, 2006.

[14] D. T. Paris, W. M. Leach Jr., and E. B. Joy, "Basic theory of probecompensated near-field measurements," IEEE Transactions on Antennas and Propagation, vol. 26, no. 3, pp. 373-379, 1978.

[15] E. B. Joy, W. M. Leach Jr., G. P. Rodrigue, and D. T. Paris, "Applications of probe-compensated near-field measurements," IEEE Transactions on Antennas and Propagation, vol. 26, no. 3, pp. 379-389, 1978.

[16] W. M. Leach Jr. and D. T. Paris, "Probe compensated near-field measurements on a cylinder," IEEE Transactions on Antennas and Propagation, vol. 21, no. 4, pp. 435-445, 1973.

[17] J. Hald, J. E. Hansen, F. Jensen, and F. H. Larsen, Spherical Nearfield Antenna Measurements, IEE Electromagnetic Waves Series, Peter Peregrinus, London, UK, 1998.

[18] P. C. Clemmov, The Plane Wave Spectrum Representation of Electromagnetic Fields, Pergamon, London, UK, 1966.

[19] D. M. Kerns, Plane-Wave Scattering Matrix Theory of Antennas and Antenna-Antenna Interactions, National Bureau of Standards Monograph no. 162, Government Printing Office, Washington, DC, USA, 1981.

[20] F. D’Agostino, C. Gennarelli, R. Guerriero, G. Riccio, and C. Savarese, "NF-FF transformation with planar wide-mesh scanning," Atti della Fondazione Giorgio Ronchi, vol. 59, pp. 781796, 2004.

[21] F. Ferrara, C. Gennarelli, R. Guerriero, G. Riccio, and C. Savarese, "An efficient near-field to far-field transformation using the planar wide-mesh scanning," Journal of Electromagnetic Waves and Applications, vol. 21, no. 3, pp. 341-357, 2007.

[22] O. M. Bucci, C. Gennarelli, and C. Savarese, "Representation of electromagnetic fields over arbitrary surfaces by a finite and nonredundant number of samples," IEEE Transactions on Antennas and Propagation, vol. 46, no. 3, pp. 351-359, 1998.

[23] O. M. Bucci and C. Gennarelli, "Application of nonredundant sampling representations of electromagnetic fields to NF-FF transformation techniques," International Journal of Antennas and Propagation, vol. 2012, Article ID 319856, 14 pages, 2012.

[24] M. A. Qureshi, C. H. Schmidt, and T. F. Eibert, "Adaptive sampling in multilevel plane wave based near-field far-field transformed planar near-field measurements," Progress in Electromagnetics Research, vol. 126, pp. 481-497, 2012.

[25] C. H. Schmidt and T. F. Eibert, "Multilevel plane wave based near-field far-field transformation for electrically large antennas in free-space or above material halfspace," IEEE Transactions on Antennas and Propagation, vol. 57, no. 5, pp. 1382-1390, 2009.

[26] F. D’Agostino, F. Ferrara, C. Gennarelli, R. Guerriero, and M. Migliozzi, "Laboratory tests assessing the effectiveness of the NF-FF transformation with helicoidal scanning for electrically long antennas," Progress in Electromagnetics Research, vol. 98, pp. 375-388, 2009. 
[27] F. D’Agostino, F. Ferrara, C. Gennarelli, R. Guerriero, and M. Migliozzi, "Experimental results validating the near-field to farfield transformation technique with helicoidal scan," The Open Electrical and Electronic Engineering Journal, vol. 4, pp. 10-15, 2010.

[28] F. D’Agostino, F. Ferrara, C. Gennarelli, G. Gennarelli, R. Guerriero, and M. Migliozzi, "On the direct non-redundant near-field-to-far-field transformation in a cylindrical scanning geometry," IEEE Antennas and Propagation Magazine, vol. 54, no. 1, pp. 130-138, 2012.

[29] F. D’Agostino, F. Ferrara, C. Gennarelli, G. Gennarelli, R. Guerriero, and M. Migliozzi, "Antenna pattern reconstruction directly from nonredundant near-field measurements collected by a cylindrical facility," Progress in Electromagnetics Research $M$, vol. 24, pp. 235-249, 2012.

[30] F. D’Agostino, F. Ferrara, C. Gennarelli, R. Guerriero, and M. Migliozzi, "Experimental assessment of an effective nearfield-far-field transformation with spherical spiral scanning for quasi-planar antennas," IEEE Antennas and Wireless Propagation Letters, vol. 12, pp. 670-673, 2013.

[31] F. D’Agostino, F. Ferrara, C. Gennarelli, R. Guerriero, and M. Migliozzi, "Far-field reconstruction from near-field data acquired via a fast spherical spiral scan: experimental evidences," Progress in Electromagnetics Research, vol. 140, pp. 719732, 2013.

[32] F. D’Agostino, F. Ferrara, J. A. Fordham, C. Gennarelli, R. Guerriero, and M. Migliozzi, "An experimental validation of the near-field-far-field transformation with spherical spiral scan," IEEE Antennas and Propagation Magazine, vol. 55, no. 3, pp. 228-235, 2013.

[33] F. D’Agostino, F. Ferrara, C. Gennarelli, R. Guerriero, and M. Migliozzi, "Non-redundant spherical NF-FF transformations using ellipsoidal antenna modellings: experimental assessments," IEEE Antennas and Propagation Magazine, vol. 55, no. 4, pp. 166-175, 2013.

[34] F. D’Agostino, F. Ferrara, C. Gennarelli, R. Guerriero, and M. Migliozzi, "Experimental testing of nonredundant near-field to far-field transformations with spherical scanning using flexible modellings for nonvolumetric antennas," International Journal of Antennas and Propagation, vol. 2013, Article ID 517934, 10 pages, 2013. 

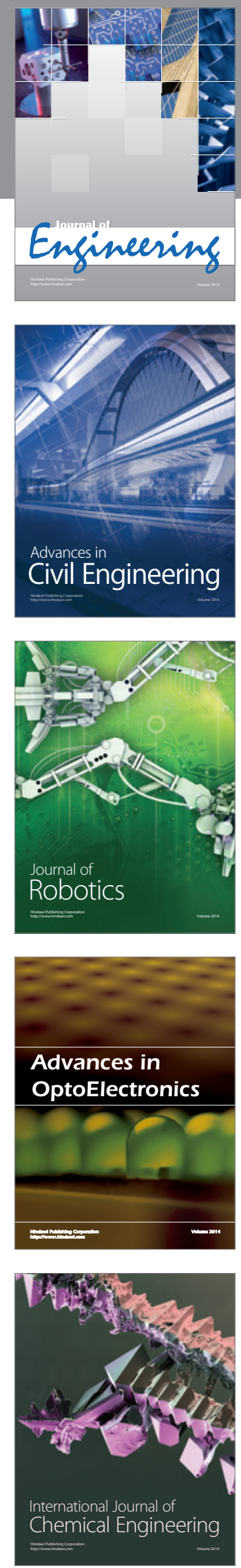

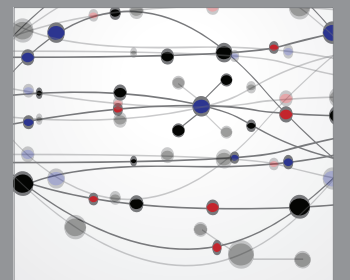

The Scientific World Journal
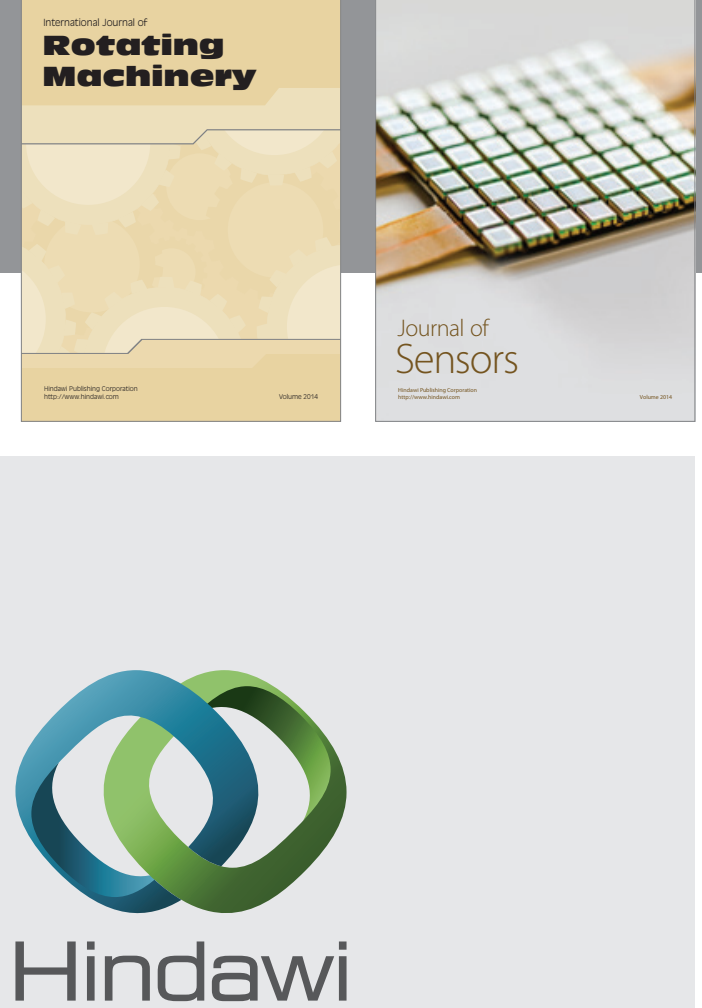

Submit your manuscripts at http://www.hindawi.com
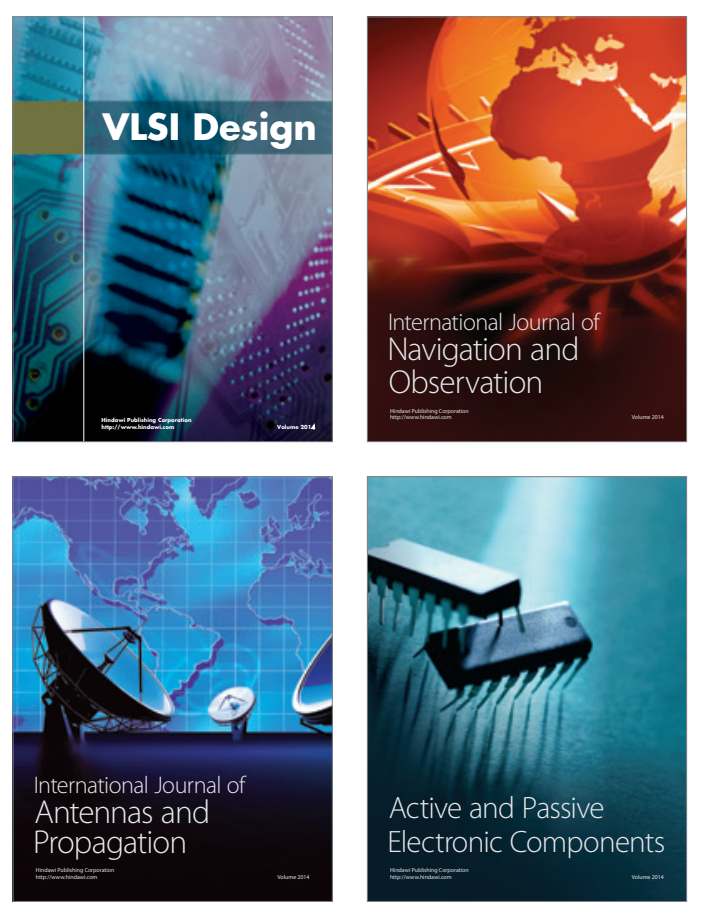
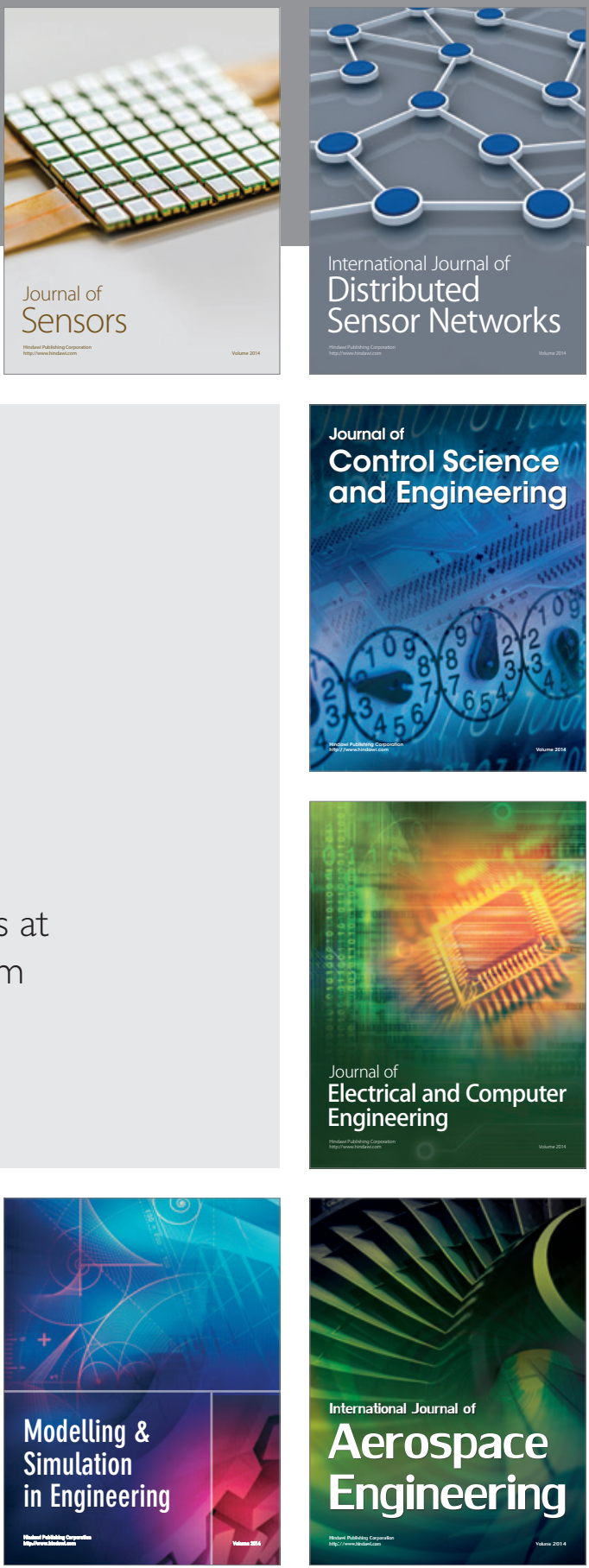

Journal of

Control Science

and Engineering
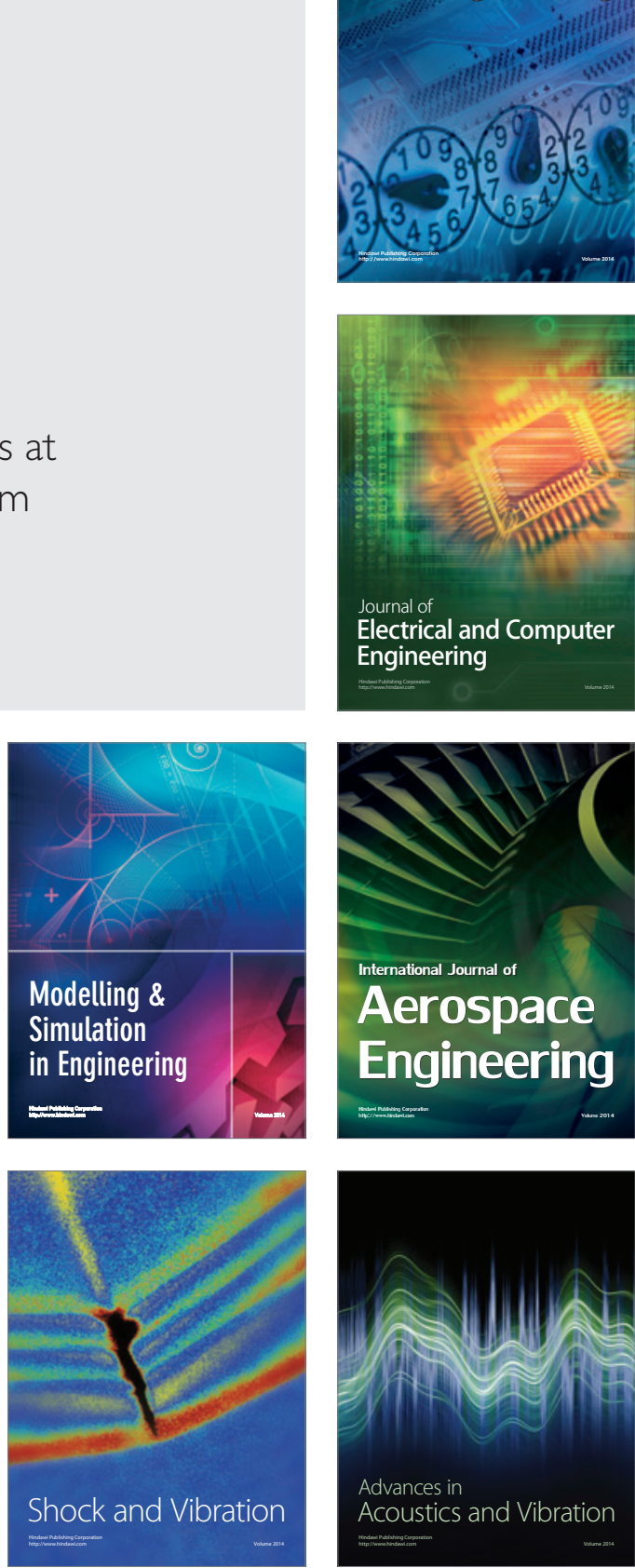